\title{
Early Results of a Novel Single-Stage Hybrid Aortic Arch Replacement Technique to Reduce Bypass and Circulatory Arrest Duration
}

\author{
Castigliano M. Bhamidipati, DO, PhD, MSc, ${ }^{1}$ Jay D. Pal, MD, $\mathrm{PhD}^{2}$ \\ ${ }^{1}$ Division of Cardiothoracic Surgery, Department of Surgery, Oregon Health and Science University, Portland, Oregon, United States; \\ ${ }^{2}$ Division of Cardiothoracic Surgery, Department of Surgery, University of Colorado, Denver, Colorado, United States
}

\section{ABSTRACT}

Objective: Hybrid repair procedures of the aortic arch have been used to reduce surgical risks and have been applied to patients who would not traditionally be candidates for open surgical repair. We present a variation on the frozen elephant trunk technique to further reduce cardiopulmonary bypass and circulatory arrest duration.

Methods: After initiation of cardiopulmonary bypass and during systemic cooling, a wire is advanced from the femoral artery into the aortic arch. In the case of aortic dissection, intravascular ultrasound is used to confirm true lumen placement. Under circulatory arrest, the proximal aortic arch is resected, and the wire is externalized. Antegrade deployment of a stent graft is performed into the aortic arch and proximal descending aorta. The ascending aortic graft is sewn to the cut end of the aorta, incorporating the stent graft. The graft is cannulated, and cardiopulmonary bypass is reinitiated. The remainder of the arch replacement is performed during rewarming. Compared with the frozen elephant trunk procedure, in which a Dacron graft is inserted into the descending aorta and later fixed with an endograft, this technique allows for immediate distal fixation. In the case of aortic dissection, there is immediate expansion of the true lumen with distal seal, potentially obviating the need for additional procedures.

Results: Twenty-two patients underwent this novel hybrid arch replacement procedure for aortic pseudoaneurysm, aortic dissection, or aneurysm. Mean duration of follow-up was 12 months (range 1-14). The mean duration of cardiopulmonary bypass was $109.32 \pm 3.14$ minutes, and mean duration of circulatory arrest was $18.00 \pm 1.33$ minutes at a mean temperature of $23.64^{\circ} \mathrm{C} \pm 0.58^{\circ} \mathrm{C}$. There were no mortalities, permanent disabling strokes, or cases of renal failure requiring dialysis.

Conclusions: This novel hybrid technique for aortic arch replacement is safe, significantly reduces cardiopulmonary bypass and circulatory arrest times, and is performed readily without need for fluoroscopy. In patients with thoracoabdominal aneurysms, the stent graft can be used as an

Received December 8, 2019; accepted December 14, 2019.

Correspondence: Castigliano M. Bhamidipati, DO, PhD, MSc, Division of Cardiothoracic Surgery, Department of Surgery, Oregon Health \& Science University, Mail Code: L353, 3181 SW Sam Fackson Park Road, Portland, OR 97239; 503-494-7820; fax: 503-494-7829 (e-mail: bhamidip@obsu.edu). elephant trunk for further thoracoabdominal aneurysm repair or branched thoracic endovascular aortic repair procedures.

\section{INTRODUCTION}

The best therapeutic options for the treatment of aortic arch aneurysms are still debated. Perioperative management and outcomes of patients is dependent on, and is influenced by, several specific operative strategies [Svensson 2004; Kouchoukos 2007; Szeto 2009; Lima 2012]. Methods to mitigate circulatory and neurological complications (eg, circulatory arrest, cooling to $18^{\circ} \mathrm{C}$, and antegrade selective cerebral perfusion) have had favorable adoption [Panos 2006; Leshnower 2010, 2013; Roselli 2010; Urbanski 2012; Pacini 2014; Angeloni 2015; Li 2016]. As such, several operative strategies, perioperative techniques, and postoperative management protocols are well described to offset morbidity and mortality [Szeto 2009; Wylie 1979; Zierer 2012; Quintana 2016; Mavroudis 2012; Di Luozzo 2012; Bavaria 1997; Wilkey 2016; Mosca 2016; Keenan 2016; Hlaing 2016; Chen 2016]. Furthermore, the advent of thoracic endovascular aortic repair (TEVAR) has resulted in favorable treatment options in aortic pathology for high-risk patients and those with prohibitive risk for open procedures [Szeto 2009; Bavaria 2013; Vallabhajosyula 2013a, b; Bavaria 2002].

Surgical methods such as the elephant trunk technique have traditionally been used to treat arch aneurysms [Svensson 2004]. These operations are complex and are impeded by their attendant risks. In an attempt to lower morbidity, increase exposure to the cardiopulmonary bypass circuit, and limit the time and need for circulatory arrest, modifications to the elephant technique have been offered [Vallabhajosyula 2013a, b]. Despite these improvements and shorter cerebral ischemia times, most modifications require the same degree of technical exposure, are fraught with a modest risk of morbidity, do not fully anatomically replicate the frozen elephant trunk technique, and do not obviate the need for an additional procedure in addressing the degenerated descending thoracic aorta.

An operation is required that (a) achieves similar results to the frozen elephant trunk technique, (b) is superior to lessinvasive approaches by definitively addressing proximal and distal thoracic aortic disease in a single stage, and (c) reduces perioperative risk. The ideal strategy for patients with aortic arch pathology (especially proximal aortic disease that 

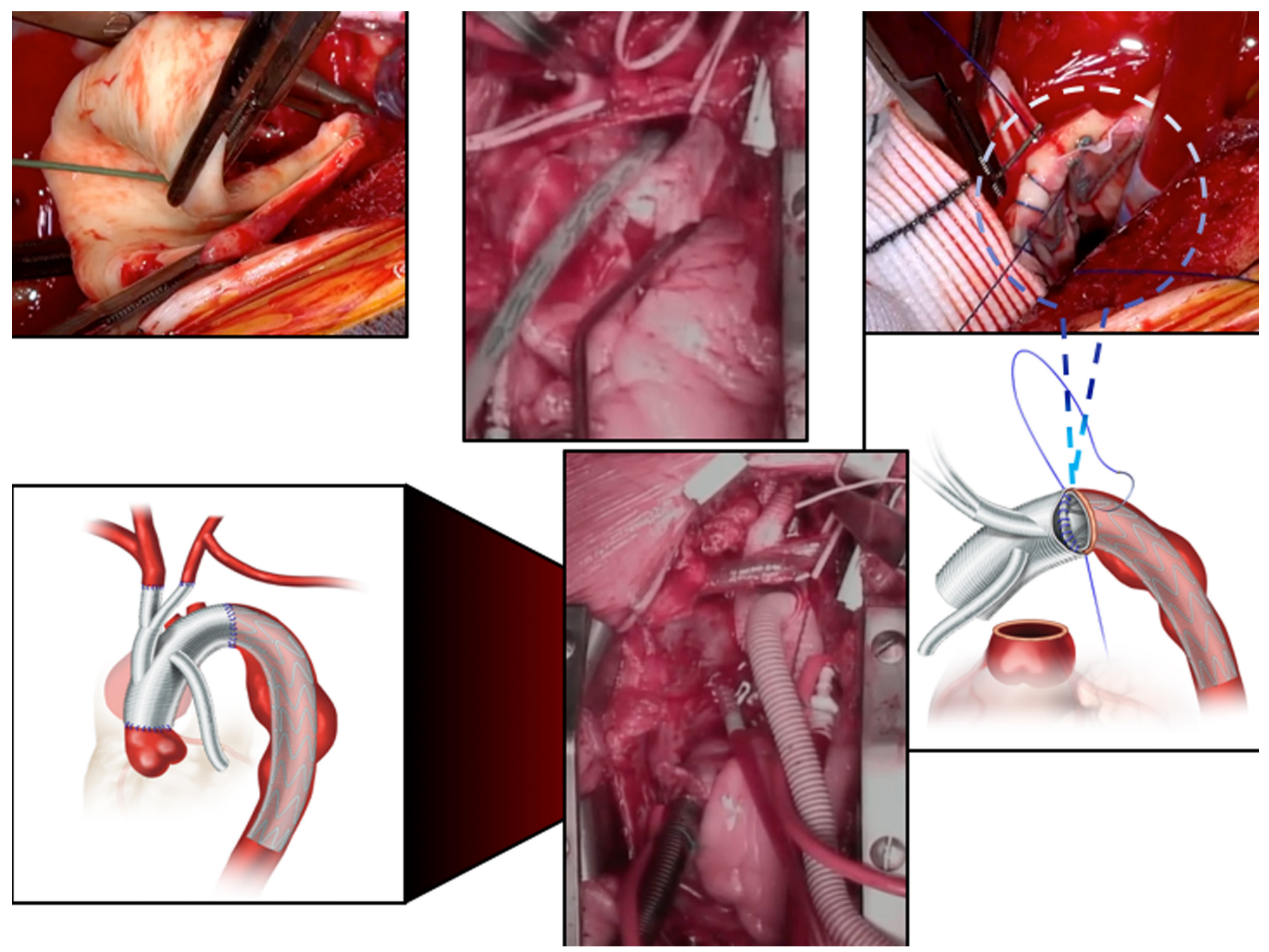

Figure 1. Tripartite suture line intussuscepts the aorta and the endograft into the branched arch. The 3 components of the suture line include the native aorta in conjunction with the Dacron multi-arm branched graft and the Dacron endoprosthetic.

progresses into the arch) is lacking. To this end, we examine our novel single-stage hybrid aortic arch replacement technique in treating various arch aortopathies, share our operative technique, and review initial results.

\section{METHODS}

\section{Preoperative Care}

All patients planned for arch surgery at our center undergo routine workup as for elective open operations, which normally includes left heart catheterization, transthoracic echocardiography, dental clearance, carotid duplex studies, medical optimization as needed, tobacco and hemoglobin A1c screening, genetic counseling when indicated, and pulmonary function testing. Preoperative planning also includes a contrast-enhanced computed tomographic scan from the aortic root through femoral arteries. In addition, all patients also undergo 3 mensio (PIE Medical Imaging
BV, Maastricht, The Netherlands) reconstruction of the entire aorta, wherein centerline measurements of the aorta are obtained. A focus on distal rather than proximal landing zones is important for our procedure. We prefer to maintain a $2-\mathrm{cm}$ distal landing zone for a good seal and normally attempt to land the endovascular thoracic stent above the diaphragm in a segment of normal aorta. We routinely use spinal cord ischemia-protective strategies as needed when longer coverage of the descending thoracic aorta is planned. Specifically, permissive hypertension, cerebrospinal fluid management, and lumbar drain are coordinated with the anesthesia and perfusion teams. Use of hybrid operating rooms or fluoroscopy is not mandatory, although availability of intravascular ultrasound (IVUS) is required.

\section{Surgical Technique}

A lumbar drain is placed in elective cases when extensive coverage of the descending thoracic aorta is planned. When the left subclavian artery is relatively posterior, a left common 


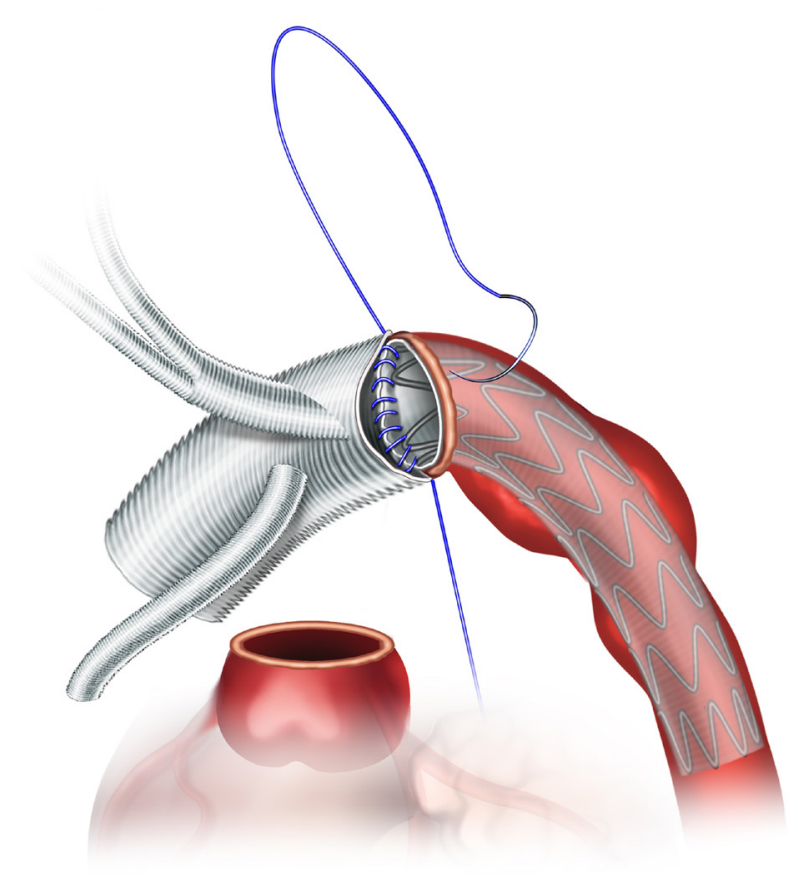

Figure 2. Completed single-stage hybrid aortic arch replacement technique. After completion of anastomosis, the connections created by this novel hybrid technique are shown.

carotid artery to left subclavian artery bypass is performed first to facilitate the arch procedure.

Standard cardiac surgery positioning and preparation are used. Bilateral cerebral oximetry monitors are placed, and right radial and femoral arterial line monitoring is maintained throughout. Circumferential dissection of the aorta from the sinotubular junction to the mid-arch at the beginning of zone 2 is accomplished. The arch vessels are also circumferentially dissected and encircled with vessel loops. The left recurrent nerve is identified and protected.

An antegrade cardioplegia catheter, distal to the vessel loop, is introduced into the innominate artery and secured for later use, as described by Jassar et al [2016]. Alternatively, a Gundry Silicone Retrograde Cardioplegia Cannula (Medtronic, Minneapolis, MN) may be introduced into the vessel lumen after transection of the innominate artery. Separately, transcutaneous ultrasound is used to obtain femoral arterial access to place an 8-French femoral sheath. An IVUS catheter is advanced through this sheath, and true lumen position is confirmed. IVUS is used to reconfirm aortic sizing and finalize device selection, typically a Valiant endoprosthesis (Medtronic) because of the ease of placing sutures through the Dacron fabric. Next, the IVUS device is removed, and the wire is held in place in the aortic arch.

Myocardial arrest with a combination of del Nido cardioplegia solution and topical cooling is achieved. The patient is systematically cooled to $20^{\circ} \mathrm{C}$ to $26^{\circ} \mathrm{C}$, depending on extent of aortic arch dissection required, and simultaneously the cardioplegia pump is used for antegrade selective cerebral

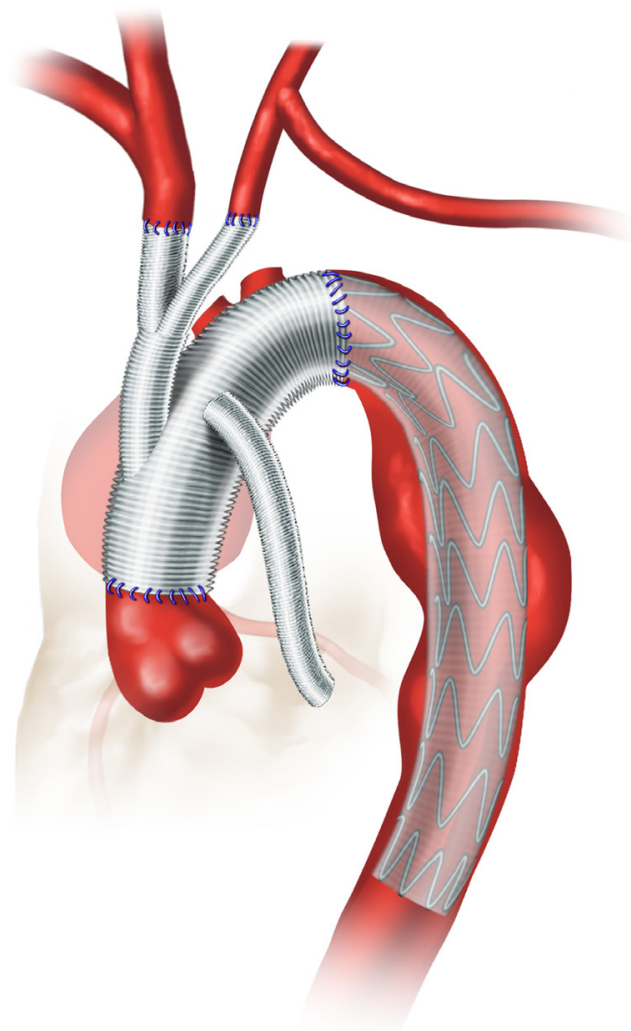

Figure 3. Composite surgical steps. This central picture captures the essence of our procedure and clearly demonstrates the key steps in this novel single-stage hybrid aortic arch replacement technique to reduce bypass and circulatory arrest duration.

perfusion. This flow rate is titrated to maintain a right radial artery pressure of $50-70 \mathrm{mmHg}$ and stable cerebral oximetry. The patient is exsanguinated, and the cardiopulmonary bypass machine is turned off. The aorta is transected at the sinotubular junction, the innominate and left common carotid arteries are clamped and disconnected from the arch, and the aorta is transected at the distal anastomosis location.

The previously placed wire is now externalized through the open aorta. This wire is used as a rail to introduce the thoracic endostent in antegrade fashion into the distal aorta. The stent is carefully deployed such that the proximal stent lands at the cut edge of the aorta. A previously measured multi-arm arch graft is used to intussuscept the aorta and the endograft into the branched arch graft such that a tripartite suture line is created (Figures 1 and 2). This maneuver creates a hybrid frozen elephant trunk. Next, the cardiopulmonary bypass circuit is attached to the side arm of the graft, and systemic antegrade flow is initiated along with systemic rewarming. The proximal anastomosis at the sinotubular junction is completed, the cross clamp is removed, the system is deaired, and myocardial perfusion is resumed. Anastomosis of the left common carotid and innominate arteries is completed, and cerebral flow is restarted (Figure 3). Weaning from cardiopulmonary bypass and closure is performed as standard for other cardiac operations. 
Table 1. Descriptive information of patients who underwent hybrid single-stage frozen elephant trunk operation

\begin{tabular}{lc}
\hline Cases $(\mathrm{n})$ & 22 \\
Mean age \pm SEM $(\mathrm{y})$ & $58.91 \pm 1.29$ \\
Male sex & $72.73 \%$ \\
Redo sternotomy & $59.09 \%$ \\
Dissection & $63.64 \%$ \\
Aneurysm & $22.73 \%$ \\
Pseudoaneurysm & $13.64 \%$
\end{tabular}

\section{Postoperative Care}

Postoperative management of the single-stage hybrid frozen elephant arch operation is similar to that of other open cardiac surgical procedures. Maintaining hemodynamic stability and permissive hypertension to minimize spinal cord ischemia is central to management. This is significant especially when greater lengths of the descending thoracic aorta are covered.

\section{Statistical Analysis}

The statistical significance of differences in proportions for categorical variables was evaluated by Pearson $\chi^{2}$ or Fisher's exact test where appropriate $(P<.05)$. The statistical significance of differences in mean values for continuous variables was assessed using single-factor analysis of variance or general linear models $(P<.05)$. Data were analyzed using Stata/IC 12.1 for Mac, revision 23 (StataCorp, College Station, TX).

\section{Quality and Process Improvement Review}

This study was approved by our institutional review board as a quality and process improvement project.

\section{RESULTS}

A total of 22 patients (mean \pm SEM age, $58.91 \pm 1.29$ years) underwent single-stage hybrid arch repair of the aortic arch aortopathy. Most patients were males (72.73\%), and almost $60 \%$ were redo sternotomy recipients. The most common indication for arch replacement was aortic dissection (>60\%), followed by ascending aneurysm $(>20 \%)$ and pseudoaneurysm (Table 1 ).

The cardiopulmonary bypass time was $109.32 \pm 3.14$ minutes, and body circulatory arrest time was 18 minutes. The cerebral ischemia time was negligible throughout the series. All patients underwent moderate hypothermic circulatory arrest to $23.64^{\circ} \mathrm{C} \pm 0.58^{\circ} \mathrm{C}$, with a mean myocardial ischemic time of 30 minutes (range 22 to 61). Most patients were extubated by the next afternoon (ventilator time, $28.48 \pm 15.71$ hours) and required nominal colloid blood product resuscitation (Table 2).

The suture line at the distal aspect of the multi-arm branched graft to freeze the elephant trunk includes 3 components, namely the endostent, the native aorta, and the Dacron
Table 2. Perioperative features of patients who underwent hybrid single-stage frozen elephant trunk operation

Cardiopulmonary bypass duration (min)
Circulatory arrest (min)
Minimum temperature $\left({ }^{\circ} \mathrm{C}\right.$ )
Myocardial ischemia (min)
Intubation (h)
Packed red blood cells (units)
Fresh-frozen plasma (units)
Platelets (units)
Cryoprecipitate (units)

$109.32 \pm 3.14$

$18.00 \pm 1.33$

$23.64 \pm 0.58$

$30.14 \pm 1.82$

$28.48 \pm 15.71$

$1.55 \pm 0.30$

$2.00 \pm 0.34$

$1.00 \pm 0.20$

$0.45 \pm 0.18$

Data are mean \pm SEM.

graft (Figure 1). The completed hybrid arch successfully addresses aortic arch pathology from the sinotubular junction through the mid-descending thoracic aorta in a single stage and is anatomically similar to the result achieved after the second stage of elephant trunk repair (Figure 2). The sequential steps and completed single-stage hybrid arch repair are shown in Figure 3.

Patients remained in the hospital for $8.36 \pm 1.10$ days; 2 patients needed tracheostomy for prolonged ventilator dependence, and 1 patient developed renal failure $(\mathrm{Cr}>2.5)$, but did not require dialysis, and postoperative infection. The vast majority of patients (>90\%) were discharged home, and 2 patients required rehabilitation (because of deconditioning) in a skilled nursing facility (Table 3 ).

\section{DISCUSSION}

Aortic arch operations are involved and require careful preoperative planning and intraoperative flexibility, meticulous hemostasis, and circulatory arrest. As such, they are challenging procedures not routinely performed at many centers. Improvements in technique, adjuncts to the operation, or modifications to postoperative care plans can be important in the effort to reduce the associated morbidity and mortality of these operations [Kouchoukos 2007; Bavaria 2013]. We review our early experience in a novel single-stage hybrid aortic arch replacement technique to reduce bypass and circulatory arrest durations. We safely offered operation for a variety of pathologies across several surgeons, including for emergent Type A dissection. Importantly, a large majority of patients were discharged home, and the related morbidity of the procedure was mitigated in several cases. This alternative technique in the management of arch pathology is performed with a hemi-arch dissection, further reducing the amount of mobilization required, while achieving a technically similar reconstruction, as seen with traditional total arch replacements and frozen elephant trunk techniques (Figure 3).

Is it well known that total arch repair requires extensive mobilization and wide exposure. Several approaches have 
Table 3. Postoperative characteristics of patients who underwent hybrid single-stage frozen elephant trunk operation

$\begin{array}{lc}\text { Mean total length of stay } \pm \text { SEM, (d) } & 8.36 \pm 1.10 \\ \text { Tracheostomy/percutaneous endoscopic gastrostomy } & 9.09 \% \\ \text { Renal failure } & 4.55 \% \\ \text { Infection } & 4.55 \% \\ \text { Home discharge } & 90.91 \%\end{array}$

been used to treat these complex patients with the use of deep hypothermic circulatory arrest through sternotomy only, left thoracotomy, bilateral anterior thoracotomy (clamshell incision), or a 2-staged approach with both sternotomy and thoracotomy (elephant trunk procedure and completion), with acceptable results. The elephant trunk technique has inherent issues: (a) it is technically challenging, especially when the true lumen is small, and (b) when the false lumen is pressurized, the elephant trunk graft could be compressed. A certain subset of patients, estimated at 10\%-15\%, die between the first and second stage of aortic repair [Kouchoukos 2007; Svensson 2008]. Others are unable to undergo the second stage of the procedure because of comorbidities. Survival is almost doubled in patients who are able to undergo the second stage of aortic repair [Svensson 2004]. Thus a surgical approach that can achieve the same anatomic effect as a total arch replacement with less exposure and complete the entirety of the aortic repair in a single stage is ideal. Our iteration of this procedure offers the flexibility of total arch replacement in a single stage, while keeping cardiopulmonary bypass and circulatory arrest times comparable to those in reports of hemiarch arch replacement [Lima 2012; Roselli 2010; Jassar 2016; Subramanian 2009].

Recently, Poletto and colleagues described a strategy to debranch arch vessels off a porcelain aorta in an octogenarian [Poletto 2016]. This amalgamated approach addressed a complex lesion. They used a combination of ventricular rapid pacing, trans-aortic approach, fluoroscopic transcatheter guidance, and an open approach, which would have normally required prolonged circulatory arrest [Poletto 2016]. Our hybrid approach uses a similar combination of open surgery and endostenting to recreate a frozen elephant trunk total arch replacement in older patients, even under emergent conditions such as acute Type A dissection. In contrast, however, our method does not require the use of hybrid operating rooms or mobile fluoroscopy. Roselli and colleagues [Lima 2012] compared their frozen elephant trunk and reversed frozen elephant trunk strategies, using the GORE® TAG® (W. L. Gore \& Associates, Flagstaff, AZ) and Zenith® TX2® (Cook Medical, Bloomington, IN) devices, in high-risk patients and found both strategies to be feasible and comparable, including in acute disease. The Cleveland Clinic experience is aggressive in the acute management of aortopathy: whereas our 2 strategies are similar, some significant differences exist. We do not use an axillary approach, and thus potentially avoid the morbidity related to brachial plexopathy, axillary hematoma, and a second surgical wound.
Proximal suture fixation to freeze the elephant trunk in situ is similar between the techniques; however, our strategy uses a single suture line between the native aorta, endostent, and ascending graft [Roselli 2010], as seen in Figures 1 and 2. Significantly, this suturing technique ensures no proximal endoleaks. Our moderate hypothermia strategy has helped keep cardiopulmonary bypass times lower and relatedly improved transfusion needs [Keenan 2016]. Taken together, these aspects show that although hybrid approaches are not novel per se, our combination and sequence of strategies has helped reduce traditionally reported cardiopulmonary bypass times by 60 minutes (in some cases) and circulatory arrest times by almost 15 minutes (depending on series).

Angeloni and colleagues [2015] recently completed a metaanalysis comparing 3,723 patients receiving bilateral antegrade selective cerebral perfusion with 3,065 patients receiving unilateral antegrade selective cerebral perfusion between 1988 and 2013. Both groups had similar postoperative mortality: bilateral patients had $9.8 \%$ mortality, and unilateral recipients, $7.6 \%$, across 32 series ranging between 17 and 673 patients [Angeloni 2015]. They also reported that both permanent and temporary neurological deficits were similar between groups (6.9\% in bilateral and 5.8\% in unilateral) [Angeloni 2015]. Importantly, longer circulatory arrest times (>40 minutes) were associated with increased mortality only in the unilateral antegrade selective perfusion group. Series size, although trended, did not significantly influence mortality or permanent stroke; moderate hypothermia was associated with the best outcomes [Angeloni 2015]. These findings further corroborate our work and are consistent across several other studies [Panos 2006; Leshnower 2010, 2013; Urbanski 2012; Pacini 2014]. Specifically, our results are favorable to the meta-regression analyses among other studies, as we only use antegrade selective circulatory perfusion titrated to radial artery perfusion pressures, our circulatory arrest times were $<25$ minutes, and we routinely use moderate-range hypothermia temperatures [Panos 2006; Leshnower 2010, 2013; Urbanski 2012; Pacini 2014; Angeloni 2015]. Our temperature strategy is supported by a propensity matched comparison in hemiarch replacement in which moderate hypothermia (median temperature $24^{\circ} \mathrm{C}$ ) was associated with slightly lower median plasma transfusion volume and chest tube output, whereas multiple other postoperative measures were similar to deep hypothermia (median temperature $17^{\circ} \mathrm{C}$ ) [Keenan 2016]. The intensive care length of stay for total arch repair recipient in our series is similar to that of 16,218 total arch replacement recipients in the Japan Adult Cardiovascular Surgery Database, in which the intensive care length of stay $>8$ days was greater in proportion among the moderate hypothermia group (47\% of cohort underwent operation at $20-25^{\circ} \mathrm{C}$ ) [Okita 2015]. Our stroke rate is low compared with other series, but is explained by differences in series size, definitions of neurologic deficits, detection rates, and variation in reporting.

\section{Limitations}

There are several clinical and reporting limitations to our work. From a clinical perspective, this is a single-center review, which is admittedly small and nascent in experience. 
Importantly, the technique is simple to replicate, and requires only some modifications. Specifically, (a) housing discretionary inventory of endostents must be available at short notice, and (b) reconfiguring of the cardiopulmonary bypass circuit lines is required to accommodate the antegrade selective cerebral flow requirements. Despite these necessities, our approach is generalizable and perhaps may be easily adopted by many centers, since the required equipment is basic and does not mandate the need for hybrid operating rooms or fluoroscopy. From a reporting perspective, we offer no comparison group, and as such our ability to discriminate outcomes or clinical features from traditional open surgery is limited. Arguably, historical controls could have been used to develop a control group; however, that approach would have been fraught with attendant criticisms of selection bias. We have used contemporaneous reports as a comparator. A priori, we believed that the effect size of major outcomes and morbidity between our method and previously described techniques was likely small, thus requiring the comparison groups to be prohibitively large. We are in the process of developing protocols for a larger multi-institutional study.

\section{Conclusions}

Aortic arch surgery includes the revascularization of the arch head vessels and myocardial and visceral protection and is completed using antegrade selective cerebral perfusion. The elephant trunk procedure, an alternative to total arch replacement, requires a second-stage operation in which the descending thoracic aorta is replaced. Several authors have offered combinations of these procedures to reduce the total number of surgeries a patient needs to undergo and in totality truncate the length of time between operations in replacing the descending thoracic aorta [Svensson 2004; Lima 2012; Roselli 2010]. In conclusion, antegrade selective cerebral perfusion, moderate hypothermia, and a single-stage singlesuture line anastomosis hybrid aortic arch replacement technique can help reduce bypass and circulatory arrest duration, to facilitate a total arch replacement with favorable outcomes.

\section{ACKNOWLEDGMENTS}

The authors collectively and sincerely thank Kate Sweeney, Medical Illustrator, University of Washington Health Sciences Academic Services \& Facilities, and the Visual Design and Production team for help with the illustrations in this manuscript.

\section{REFERENCES}

Angeloni E, Melina G, Refice SK,et al. 2015. Unilateral versus bilateral antegrade cerebral protection during aortic surgery: An updated metaanalysis. Ann Thorac Surg 99:2024-2031.

Bavaria JE, Brinster DR, Gorman RC, Woo YJ, Gleason T, Pochettino A. 2002. Advances in the treatment of acute type A dissection: An integrated approach. Ann Thorac Surg 74:S1848-S1852.

Bavaria JE, Pochettino A. 1997. Retrograde cerebral perfusion (RCP) in aortic arch surgery: efficacy and possible mechanisms of brain protection. Semin Thorac Cardiovasc Surg 9:222-232.

Bavaria J, Vallabhajosyula P, Moeller P, Szeto W, Desai N, Pochettino A. 2013. Hybrid approaches in the treatment of aortic arch aneurysms: Postoperative and midterm outcomes. J Thorac Cardiovasc Surg 145(3 suppl):S85-S90.

Chen EP, Leshnower BG. 2016. Temperature management for aortic arch surgery. Semin Cardiothorac Vasc Anesth 20:283-288.

Di Luozzo G, Griepp RB. 2012. Cerebral protection for aortic arch surgery: Deep hypothermia. Semin Thorac Cardiovasc Surg 24:127-30.

Hlaing M. 2016. Immediate ICU care for patients following aortic arch surgery. Semin Cardiothorac Vasc Anesth 20:333-342.

Jassar AS, Vallabhajosyula P, Bavaria JE, et al. 2016. Direct innominate artery cannulation: An alternate technique for antegrade cerebral perfusion during aortic hemiarch reconstruction. J Thorac Cardiovasc Surg 151:1073-1078.

Keenan JE, Benrashid E, Kale E, Nicoara A, Husain AM, Hughes GC. 2016. Neurophysiological intraoperative monitoring during aortic arch surgery. Semin Cardiothorac Vasc Anesth 20:273-282.

Keenan JE, Wang H, Gulack BC, et al. 2016. Does moderate hypothermia really carry less bleeding risk than deep hypothermia for circulatory arrest? A propensity-matched comparison in hemiarch replacement. J Thorac Cardiovasc Surg 152:1559-1569.e2.

Kouchoukos NT, Mauney MC, Masetti P, Castner CF. 2007. Optimization of aortic arch replacement with a one-stage approach. Ann Thorac Surg 83:S811-S814

Leshnower BG, Myung RJ, Chen EP. 2013. Aortic arch surgery using moderate hypothermia and unilateral selective antegrade cerebral perfusion. Ann Cardiothorac Surg 2:288-295.

Leshnower BG, Myung RJ, Kilgo PD, et al. 2010. Moderate hypothermia and unilateral selective antegrade cerebral perfusion: a contemporary cerebral protection strategy for aortic arch surgery. Ann Thorac Surg 90:547-554.

Li Y, Siemeni T, Optenhoefel J, et al. 2016. Pressure level required during prolonged cerebral perfusion time has no impact on neurological outcome: A propensity score analysis of 800 patients undergoing selective antegrade cerebral perfusion. Interact Cardiovasc Thorac Surg 23:616-622.

Lima B, Roselli EE, Soltesz EG, et al. 2012. Modified and "reverse" frozen elephant trunk repairs for extensive disease and complications after stent grafting. Ann Thorac Surg 93:103-109.

Mavroudis CD, Molina E, Stewart A. 2012. Cerebral protection for aortic arch surgery: Hybrid approach. Semin Thorac Cardiovasc Surg 24:302-304.

Mosca MS, Justison G, Reece TB. 2016. A clinical protocol for goal directed cerebral perfusion during aortic arch surgery. Semin Cardiothorac Vasc Anesth 20:289-297.

Okita Y, Miyata H, Motomura N, Takamoto S. 2015. A study of brain protection during total arch replacement comparing antegrade cerebral perfusion versus hypothermic circulatory arrest, with or without retrograde cerebral perfusion: Analysis based on the Japan Adult Cardiovascular Surgery Database. J Thorac Cardiovasc Surg 149(2 suppl):S65-S73.

Pacini D, Pantaleo A, Di Marco L, et al. 2014. Visceral organ protection in aortic arch surgery: Safety of moderate hypothermia. Eur J Cardiothorac Surg 46:438-443. 
Panos A, Murith N, Bednarkiewicz M, Khatchatourov G. 2006. Axillary cerebral perfusion for arch surgery in acute type A dissection under moderate hypothermia. Eur J Cardiothorac Surg 29:1036-1039.

Poletto GL, Musto L, Civilini E, Giorgetti P. 2016. Clampless and sutureless hybrid technique for aortic arch debranching on a porcelain aorta. Ann Thorac Surg 101:2395-2397.

Quintana E, Bajona P, Schaff HV, et al. 2016. Open aortic arch reconstruction after coronary artery bypass surgery: Worth the effort? Semin Thorac Cardiovasc Surg 28:26-35.

Roselli EE, Soltesz EG, Mastracci T, Svensson LG, Lytle BW. 2010. Antegrade delivery of stent grafts to treat complex thoracic aortic disease. Ann Thorac Surg 90:539-546.

Subramanian S, Roselli EE. 2009. Thoracic aortic dissection: Long-term results of endovascular and open repair. Semin Vasc Surg 22:61-68.

Svensson LG, Kim KH, Blackstone EH, et al. 2004. Elephant trunk procedure: Newer indications and uses. Ann Thorac Surg 78:109-116.

Svensson LG, Kouchoukos NT, Miller DC, et al. 2008. Expert consensus document on the treatment of descending thoracic aortic disease using endovascular stent-grafts. Ann Thorac Surg 85(1 suppl):S1-S41.
Szeto WY, Bavaria JE. 2009. Hybrid repair of aortic arch aneurysms: Combined open arch reconstruction and endovascular repair. Semin Thorac Cardiovasc Surg 21:347-354.

Urbanski PP, Lenos A, Bougioukakis P, Neophytou I, Zacher M, Diegeler A. 2012. Mild-to-moderate hypothermia in aortic arch surgery using circulatory arrest: A change of paradigm? Eur J Cardiothorac Surg 41:185-191.

Vallabhajosyula P, Szeto W, Desai N, Bavaria JE. 2013. Type I and type II hybrid aortic arch replacement: Postoperative and mid-term outcome analysis. Ann Cardiothorac Surg 2:280-287.

Vallabhajosyula P, Szeto WY, Desai N, Komlo C, Bavaria JE. 2013. Type II arch hybrid debranching procedure. Ann Cardiothorac Surg 2:378-386

Wilkey BJ, Weitzel NS. 2016. Anesthetic considerations for surgery on the aortic arch. Semin Cardiothorac Vasc Anesth 20:265-272.

Wylie EJ, Effeney DJ. 1979. Surgery of the aortic arch branches and vertebral arteries. Surg Clin North Am 59:669-680.

Zierer A, Moritz A. 2012 Cerebral protection for aortic arch surgery: Mild hypothermia with selective cerebral perfusion. Semin Thorac Cardiovasc Surg 24:123-126. 